\title{
AN INTRINSIC MECHANISM OF CORTICOGENESIS FROM EMBRYONIC STEM CELLS
}

Nicolas Gaspard ${ }^{1}$, Tristan Bouschet ${ }^{1}$, Raphael Hourez ${ }^{2}$, Jordane Dimidschstein ${ }^{1}$, Gilles Naeije $^{1}$, Jelle van den Ameele ${ }^{1}$, Ira Espuny-Camacho ${ }^{1}$, Adèle Herpoel ${ }^{1}$, Lara Passante ${ }^{1}$, Serge N. Schiffmann ${ }^{2}$, Afsaneh Gaillard ${ }^{3}$ \& Pierre Vanderhaeghen ${ }^{1}$

${ }^{1}$ IRIBHM (Institute for Interdisciplinary Research), ${ }^{2}$ Laboratory of Neurophysiology, Université Libre de Bruxelles (ULB), 808 Route de Lennik, B-1070 Brussels, Belgium. Institut de Physiologie et Biologie Cellulaires, Université de Poitiers, Centre National de la Recherche Scientifique (CNRS), 40 avenue du recteur Pineau, Poitiers, F-86022, France.

\begin{abstract}
The cerebral cortex develops through the coordinated generation of dozens of neuronal subtypes, but the mechanisms involved remain unclear. Here we show that mouse embryonic stem cells, cultured without any morphogen but in the presence of a sonic hedgehog inhibitor, recapitulate in vitro the major milestones of cortical development, leading to the sequential generation of a diverse repertoire of neurons that display most salient features of genuine cortical pyramidal neurons. When grafted into the cerebral cortex, these neurons develop patterns of axonal projections corresponding to a wide range of cortical layers, but also to highly specific cortical areas, in particular visual and limbic areas, thereby demonstrating that the identity of a cortical area can be specified without any influence from the brain. The discovery of intrinsic corticogenesis sheds new light on the mechanisms of neuronal specification, and opens new avenues for the modelling and treatment of brain diseases.
\end{abstract}


The cerebral cortex is the most complex structure in the mammalian brain, and displays an unparalleled diversity of neuronal subtypes. This complexity is established sequentially, starting with the emergence of the cortical primordium in the forebrain. Forebrain identity is thought to constitute a primitive pattern of neural identity, which is retained through the inhibition of caudalizing morphogen signals ${ }^{1}$. The forebrain then undergoes patterning along the dorso-ventral axis, mainly through the induction of ventral identity by sonic hedgehog $(\mathrm{SHH})$, secreted from the ventral neural tube and underlying tissue ${ }^{2}$. This leads to the specification of the two main populations of cortical neurons, pyramidal neurons and interneurons, which are generated from the dorsal and the ventral part of the telencephalon, respectively ${ }^{1,3-5}$.

A next level of complexity emerges through the specification of different subtypes of cortical neurons that will populate specific cortical layers, where they exhibit specific patterns of gene expression and connectivity. This specification follows a coordinated temporal pattern: neurons from different layers are generated sequentially ${ }^{6}$, but the underlying mechanisms remain poorly known ${ }^{7-10}$. Finally, neurons from distinct cortical areas develop selective patterns of gene expression and projections, through the interplay between factors intrinsic to the cortex and extrinsic factors from the brain and body $y^{3,11-13 .}$

A better understanding of cortical development would greatly benefit from reductionist approaches. Here we tested whether the complex developmental processes leading to the formation of the cerebral cortex could be studied in a meaningful way using an embryonic stem cell (ESC)-based system. This led to the surprising discovery of an intrinsic pathway that recapitulates in vitro most major milestones of corticogenesis.

\section{GENERATING CORTICAL PROGENITORS FROM ESCS}

To explore the possibility that forebrain identity might emerge during differentiation of ESCs in default conditions, as during in vivo embryogenesis ${ }^{1}$, we adapted a method of neurogenesis from adherent monocultures of ESCs ${ }^{14}$. We cultured ESCs at low density in a chemically defined default medium (DDM) devoid of serum or any morphogen but allowing cell survival by insulin. ESCs underwent an efficient neurogenesis process leading first to the generation of nestin ${ }^{+}$neural progenitors, followed by $\beta$-tubulin-III ${ }^{+}$and $\mathrm{MAP}^{+}{ }^{+}$(also known as MTAP $^{+}$) neurons, and finally by astroglial production (Fig. 1a and Supplementary Fig. 1). Thus, ESCs cultured in the absence of any added morphogen efficiently differentiate into neural cells, as described ${ }^{15,16}$, and follow a neuron-glia sequence highly reminiscent of cortical cells in vitro and in vivo ${ }^{17}$.

We next looked at the regional identity of the neural progenitors generated in these default conditions: we found that most neural precursors expressed early markers of the anterior neurectoderm (OTX1 and OTX2, Fig. 1c, n), markers of the dorsal forebrain (OTX1 and PAX6, Fig. 1d, e, $n$ ) and to a larger extent markers of the ventral forebrain (GSH2 (also known as GSX2) and NKX2-1, Fig. 1f, g, n and Supplementary Fig. 2a, d). In contrast, only a minority $(<1 \%)$ of progenitors expressed markers indicative of more caudal identity (Fig. 1n and Supplementary Fig. 2b, c). The fore-brain-like identity of ESC-derived neural progenitors was further confirmed by polymerase chain reaction with reverse transcription (RT-PCR) experiments (Fig. 1m). 
Because $\mathrm{SHH}$ is required for the induction of the ventral identity of the forebrain, we hypothesized that the large proportion of ventral forebrain progenitors in DDM cultures might be induced by an endogenous $\mathrm{SHH}$ activity. Consistent with this hypothesis, we found that Shh was induced after 4 days of DDM culture (Fig. $1 \mathrm{~m}$ ). We then attempted to block it during the neural progenitor differentiation process by using cyclopamine, a specific antagonist of the SHH pathway ${ }^{18}$ (referred to as the 'DDM plus cyclopamine' condition). Cyclopamine did not change the neuralization process, nor did it alter the forebrain identity of the neural progenitors (Fig. 1a, b, h, n) or their proliferative pattern (data not shown). However, the addition of cyclopamine caused a massive increase in the expression of dorsal markers, whereas expression of ventral markers was almost abolished (Fig. 1i-n; $P<0.001$ for all markers). Thus, after inhibition of SHH signalling in DDM cultures, ESCs can be converted to a largely homogeneous population of neural progenitors expressing markers of the presumptive cerebral cortex (Supplementary Fig. $2 e)^{19}$.

\section{Figure 1. Differentiation of ESCs into cortical progenitors, $a, b$}

Sequential generation of nestin ${ }^{+}$neural progenitors, $\beta$-tubulin- $\mathrm{II}^{+}$neurons and $\mathrm{GFAP}^{+}$astroglial cells, after differentiation in DDM (a) or DDM plus cyclopamine (b). Histograms show the proportion of cells expressing the given marker among all cells visualized by nuclear staining. No difference is observed between DDM and DDM plus cyclopamine conditions. C-I, Expression of the early forebrain regionalization markers OTX1 and OTX2 (c, e, $\mathbf{h}, \mathbf{j})$, PAX6 (d, i), GSH2 (f, k) and NKX2-1 (g, l) among neural progenitors after 14 days of differentiation in DDM (c-g) or DDM plus cyclopamine (h-l). Nestin, green; markers, red; nuclear staining, blue. $\mathbf{m}$, RT-PCR of specific marker genes of the telencephalon (Foxg1), dorsal forebrain (Emx1 and Emx2) and ventral forebrain (DIx1, DIx5, Nkx2-1 and Lhx6), and Shh from day 4-14 cultures of cells in DDM or DDM plus cyclopamine. $\mathbf{n}$, Proportions of nestin neural progenitors expressing specific neural regionalization markers afterl4 days of differentiation in DDM or DDM plus cyclopamine, showing a conversion from ventral to dorsal forebrain identity in DDM plus cyclopamine. Data are represented as mean + s.e.m. Scale bars, $20 \mu \mathrm{m}$. 
a

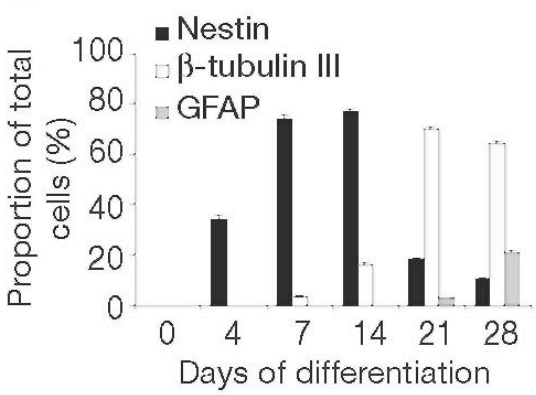

$\frac{5}{\frac{5}{0}}$
$\frac{\overline{0}}{0}$
$\frac{0}{0}$
$\stackrel{0}{\frac{0}{4}}$
$\frac{0}{7}$

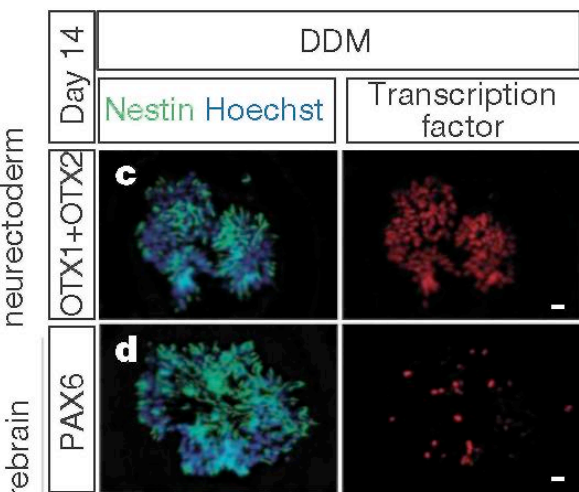

造
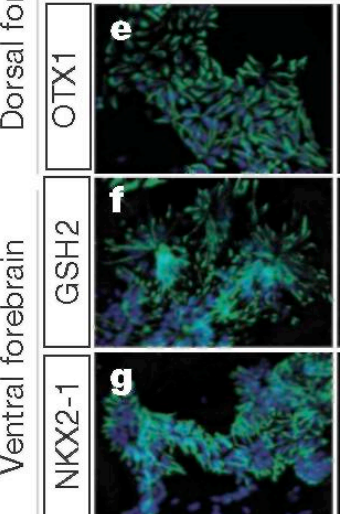

m
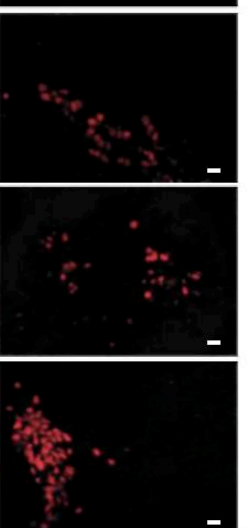

$\mathrm{DDM}+$ DDM cyclopamine
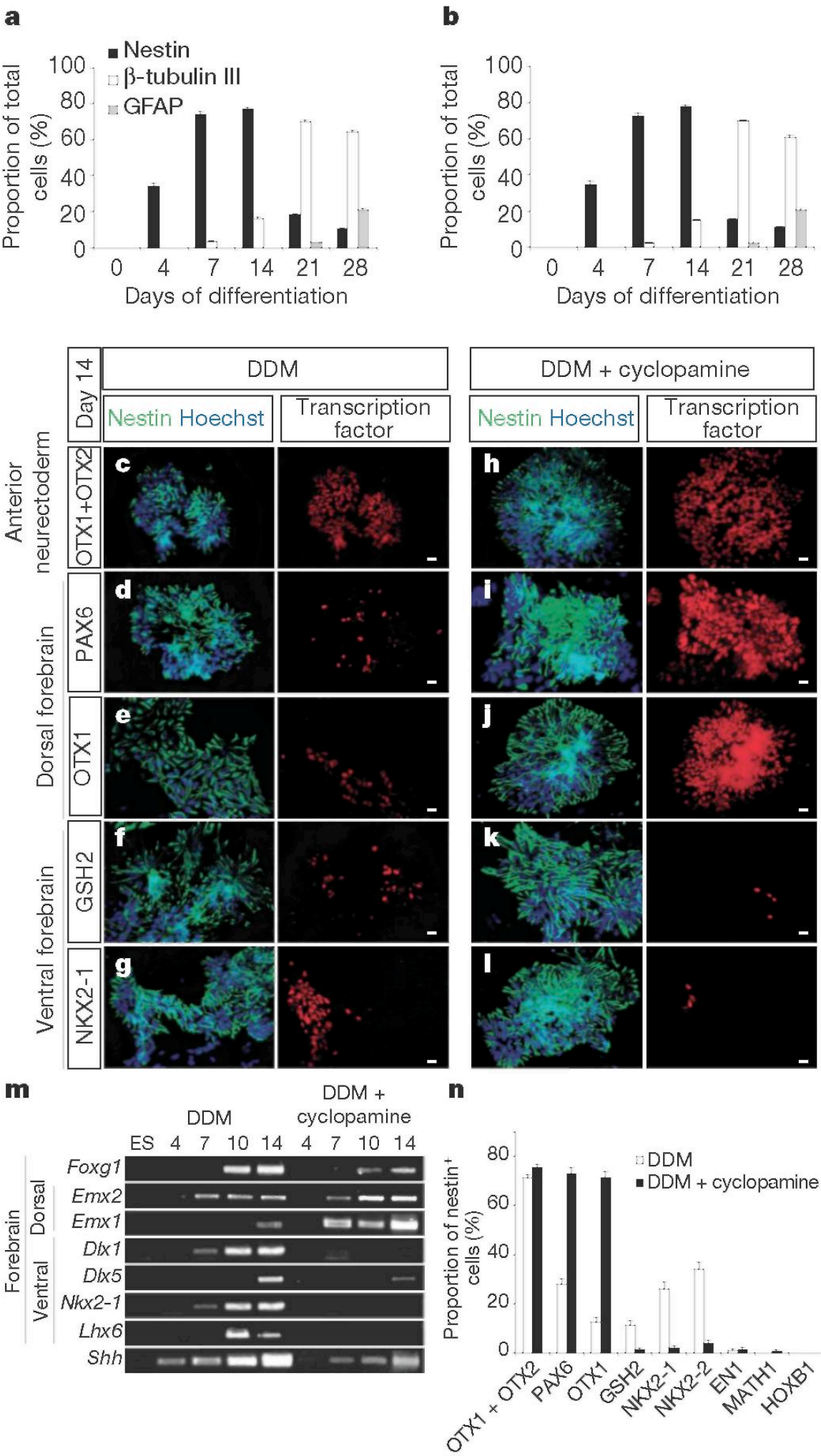

n 


\section{Figure 2. Generation of functional cortical neurons from ESCs in DDM plus cyclopamine. a-f}

Expression of glutamatergic vesicular transporters VGLUT1 and VGLUT2 as well as the GABA vesicular transporter VGAT in $\beta$-tubulin-III ${ }^{+}$neurons after 28 days in culture, after differentiation in DDM (a-c) or DDM plus cyclopamine (d-f). Markers, red; $\beta$-tubulin- III $^{+}$(stained with Tuj1), green. Scale bar, $20 \mu \mathrm{m}$. g, Proportion of VGLUT1, VGLUT2 and VGAT expression among $\beta$-tubulin-III ${ }^{+}$neurons after 28 days in culture, in DDM or DDM plus cyclopamine conditions. Data are shown as mean + s.e.m. $\left(N=3\right.$ experiments; ${ }^{* \star *} P<0.001, z$-test for proportions), h, i, Recorded iPSCs (h) and ePSCs (i) from neurons after 28 days, after differentiation in DDM, showing inhibition by picrotoxin (GABAA antagonist) and APV/NBQX (NMDA and AMPA receptors antagonists), respectively. The second EPSC in $i$ is the EPSC marked with an asterisk in the first graph shown at a different time scale, j, Proportion of recorded neurons displaying iPSCs and ePSCs in each condition. Data are shown as mean + s.e.m. $(N=33$ neurons; $P<0.05$, Fischer exact t-test). ACSF: artificial cerebrospinal fluid.

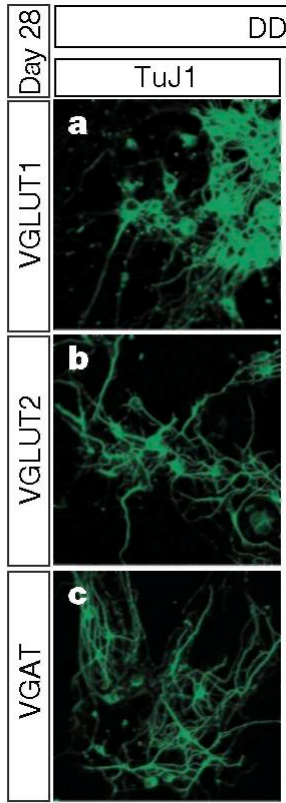

$\mathbf{g}$

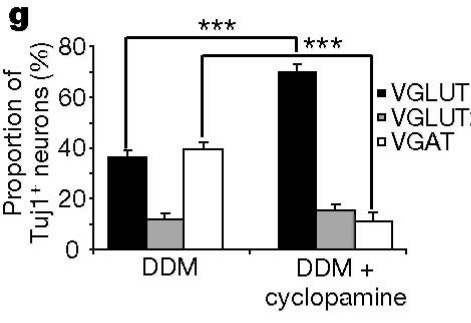

I ACSF $+100 \mu \mathrm{M}$ picrotoxin $-60 \mathrm{mV}$

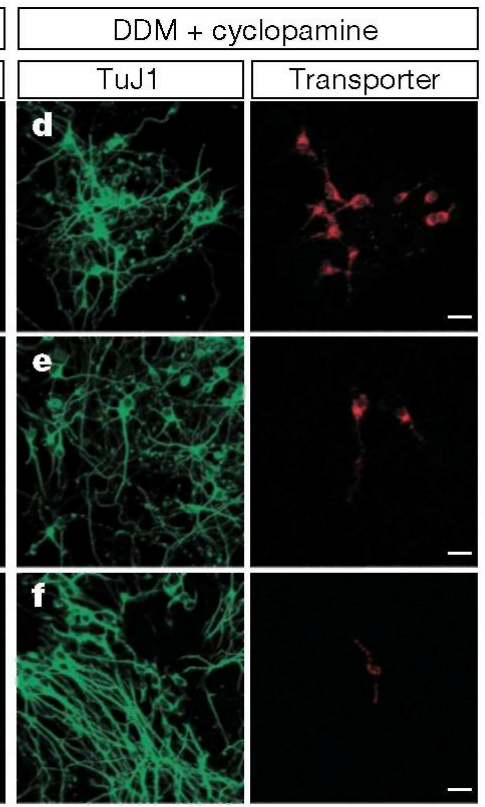

h $\mathrm{ACSF}+50 \mu \mathrm{M}$ APV and $50 \mu \mathrm{M}$ NBQX $-20 \mathrm{mV}$

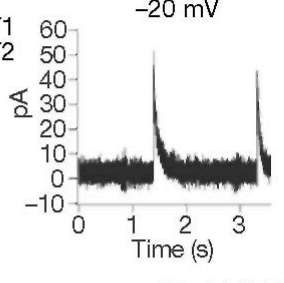

$+50 \mu \mathrm{M}$ APV and $5 \mu \mathrm{M}$ NBQX

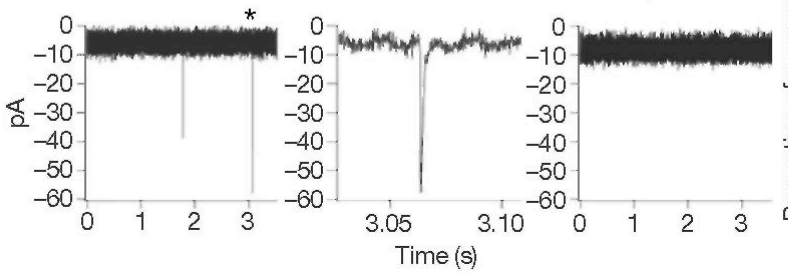

$+100 \mu \mathrm{M}$ picrotoxin
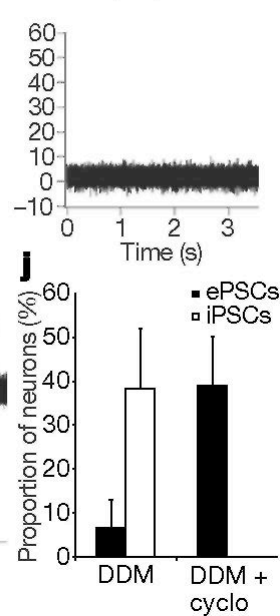


\section{GENERATING PYRAMIDAL NEURONS FROM ESCS}

We next examined the identity of the neurons generated in DDM and DDM plus cyclopamine conditions. We found that most $\beta$-tubulin- $\mathrm{II}^{+}$neurons derived from neural progenitors generated in DDM plus cyclopamine expressed the vesicular glutamate transporters VGLUT1 and VGLUT2 (also known as SLC17A7 and SLC17A6, respectively, Fig. 2d), markers of pyramidal neurons of the cerebral cortex (Fig. 2e), whereas only a minority of them expressed the vesicular GABA ( $\gamma$-aminobutyric acid) transporter VGAT, a marker of GABAergic inhibitory neurons (Fig. 2f). In the case of neurons generated in DDM, we observed a much greater proportion of GABAergic neurons (Fig. 2a-g; $P<0.001$, z-test for proportions) and a much lower proportion of glutamatergic neurons $(P<0.001$ and $P>0.05$, respectively, for VGLUT1 and VGLUT2). No markers of non-cortical fates, such as tyrosine hydroxylase, choline acetyltransferase, rhodopsin or GABA-a6-receptor were found in either DDM or DDM plus cyclopamine conditions (data not shown). We then performed patchclamp recordings, and found that neurons showed spontaneous action potentials and synaptic currents in both DDM and DDM plus cyclopamine conditions (Fig. $2 \mathrm{~h}$, i and Supplementary Fig. 3a-i). However, in DDM conditions, most recorded neurons displayed spontaneous GABAergic inhibitory post-synaptic currents (iPSCs), whereas, in DDM plus cyclopamine conditions, most recorded neurons displayed glutamatergic excitatory postsynaptic currents (ePSCs; Fig. 2j; $\mathrm{P}<0.05$; Fisher exact $t$ - test). Thus, the differences in expression of neurotransmission markers between neurons derived from DDM and those from DDM plus cyclopamine conditions correlate with functional differences in synaptic activity.

Cortical neurons can be also distinguished by their morphological properties: cortical excitatory neurons exhibit a pyramidal or unipolar morphology, characterized by the presence of one dendrite that is wider than the others, whereas GABAergic interneurons show multipolar morphologies. We compared the morphology of the neurons generated in each condition, and observed an important qualitative shift, with many more neurons in DDM plus cyclopamine displaying a unipolar pyramidal morphology (Fig. 3a-c, $\mathrm{P}<0.01, X^{2}$ test). We used a pyramidal morphology index $(\mathrm{PMI})^{20}$ to objectively distinguish the dendritic morphology of cortical pyramidal neurons and inhibitory neurons. Most neurons generated in DDM plus cyclopamine displayed a PMI corresponding to pyramidal neurons that was significantly higher than that in DDM conditions (Fig. 3d; $P<0.001$, z-test for proportions; Supplementary Fig. 4; $\mathrm{P}<0,001$; Mann-Whitney test).

Within the cortex, glutamatergic pyramidal neurons are polarized and oriented radially, with one large dendrite pointing towards the cortical surface and the axon pointing towards the ventricular surface. Using a slice overlay assay ${ }^{21}$ we tested whether ESC-derived neurons can orient radially like pyramidal neurons. Neurons were first differentiated in vitro from green fluorescent protein (GFP) ESCs, and then dissociated and plated on mouse postnatal cortical slices. Most neurons generated in DDM plus cyclopamine displayed a radial orientation with a dendrite pointing to the cortical surface (Fig. 3e-h), whereas only a minor fraction of neurons generated in DDM showed this behaviour (Fig. 3h; $\mathrm{P}<0,001$ ).

Together, these data indicate that most of the neurons generated from ESCs in DDM plus cyclopamine display morphological and functional properties characterizing pyramidal neurons, whereas neural progenitors produced in DDM produces a mixture of GABAergic 
multipolar neurons and pyramidal neurons, in marked correlation with the differential dorsoventral identity of the neural progenitors generated in each condition.

\section{Figure 3. ESC-derived neurons in DDM plus cyclopamine display morphological features of pyramidal neurons, $a, b$}

Typical morphology observed for MAP2 ${ }^{+}$mature neurons cultured for 28 days, after differentiation in DDM (a) or DDM plus cyclopamine (b). Red arrowheads point to large dendrites that are characteristic of pyramidal neurons. Scale bar represents $20 \mu \mathrm{m}$. c, Distribution of neuron morphology. Semiquantitative scoring of neurons displaying pyramidal, unipolar or multipolar morphology $\left(N=3\right.$ experiments; $P<0.001 x^{2}$ test). d, Proportion of neurons displaying a PMI above the cut off of 1.2 after DDM or DDM plus cyclopamine conditions, showing a conversion to a pyramidal morphology in DDM plus cyclopamine $\left(N=3\right.$ experiments; ${ }^{* * *} P<0.001$, z-test for proportions). Orientation pattern of neurons derived from GFP ESCs in DDM plus cyclopamine (e-h) conditions, differentiated for 21 days, and cultured for 4 days on top of postnatal cortical slices. GFP is in white (e) and green (f) and MAP2 in red in $\mathbf{f}$. Apical surface of the cortical slices is up. Dendrites that are located apically and oriented radially are marked by red arrowheads in e. $\mathbf{g}, \mathbf{h}$, Quantification of the proportion of neurons displaying radial orientation for neurons derived in DDM or DDM plus cyclopamine conditions. Data are represented as mean + s.e.m. $(N=3$ experiments; ${ }^{* \star} P<0.001, z$-test for proportions). Scale bar represents $20 \mu \mathrm{m}$. All data are shown as mean + s.e.m. 

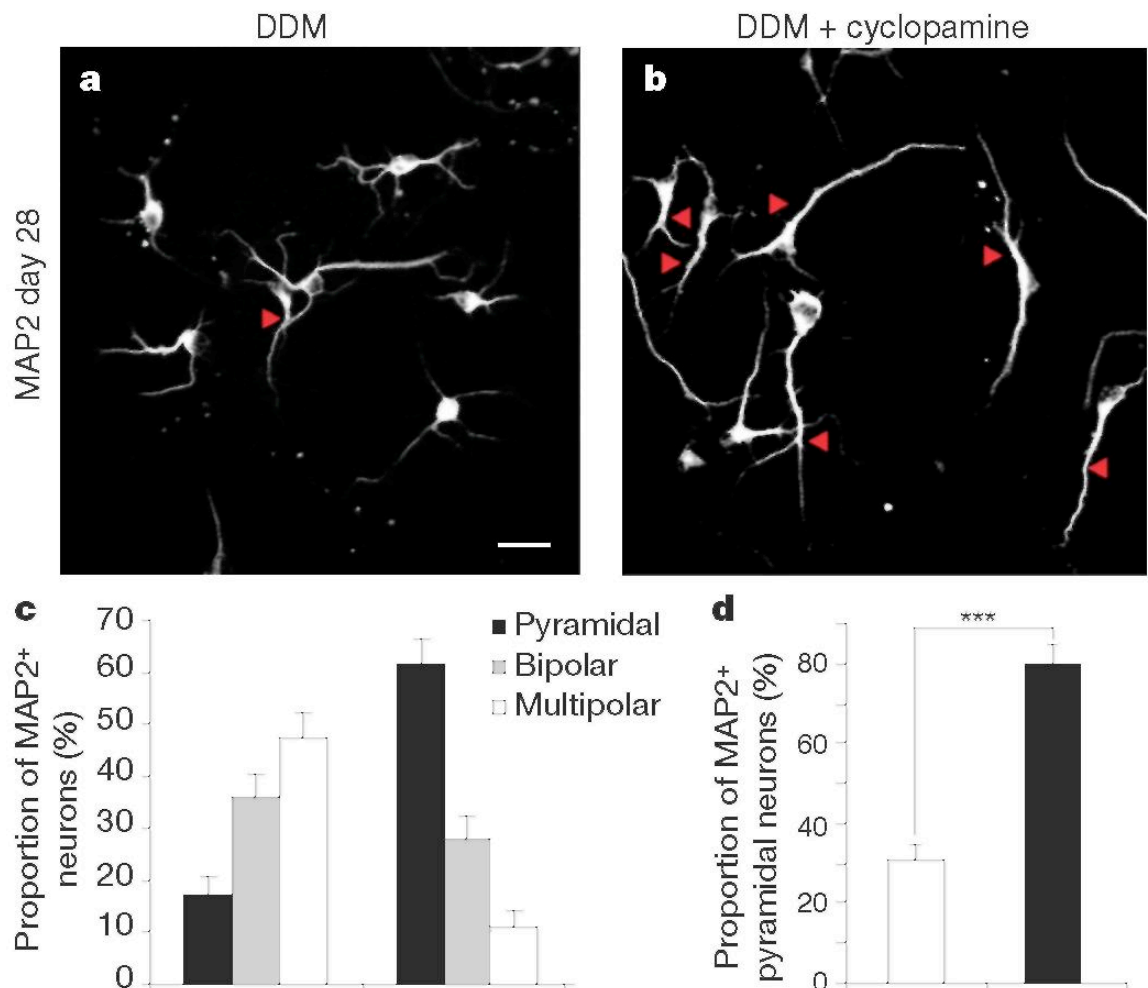

- Pyramidal
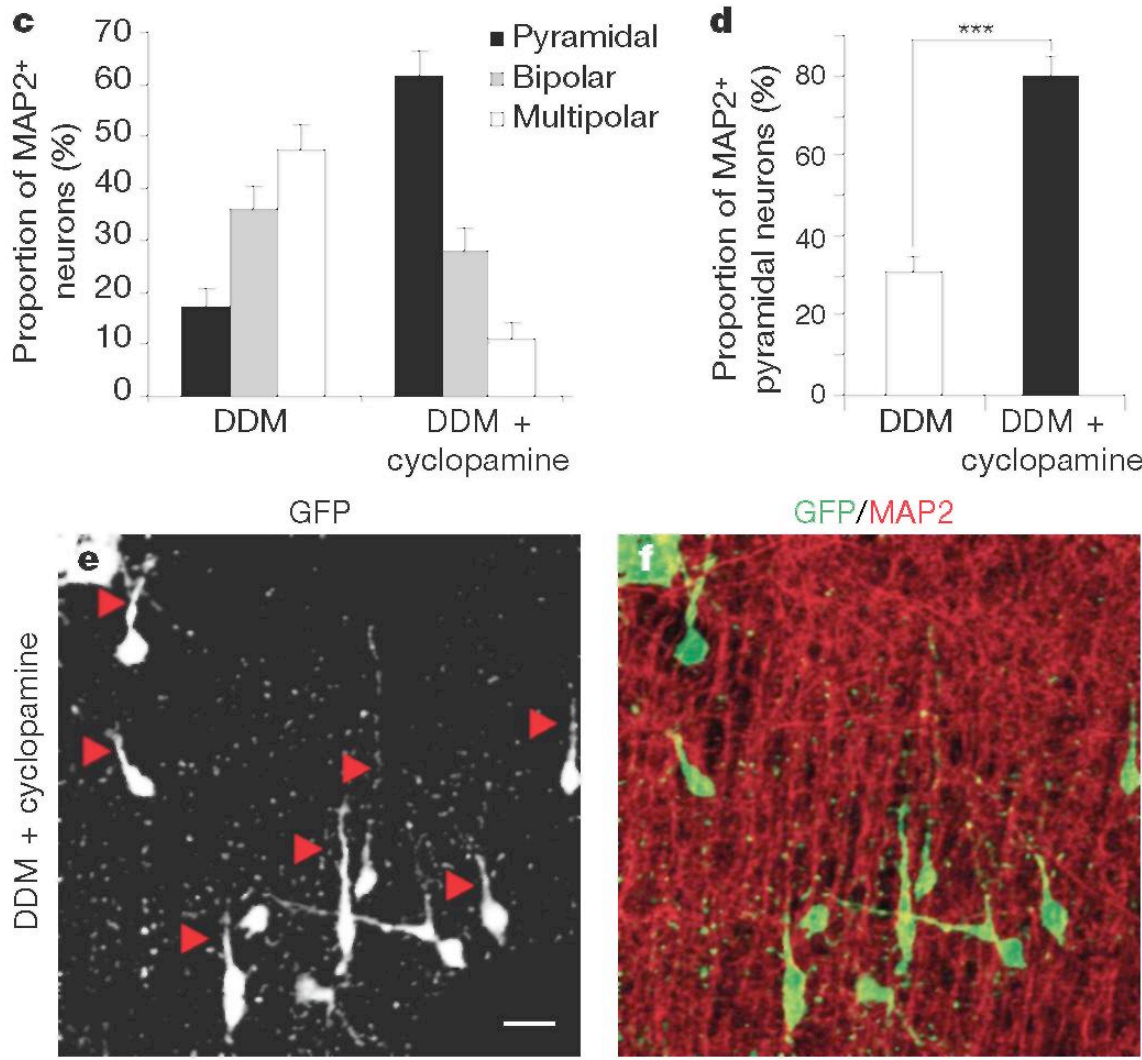

GFP/MAP2
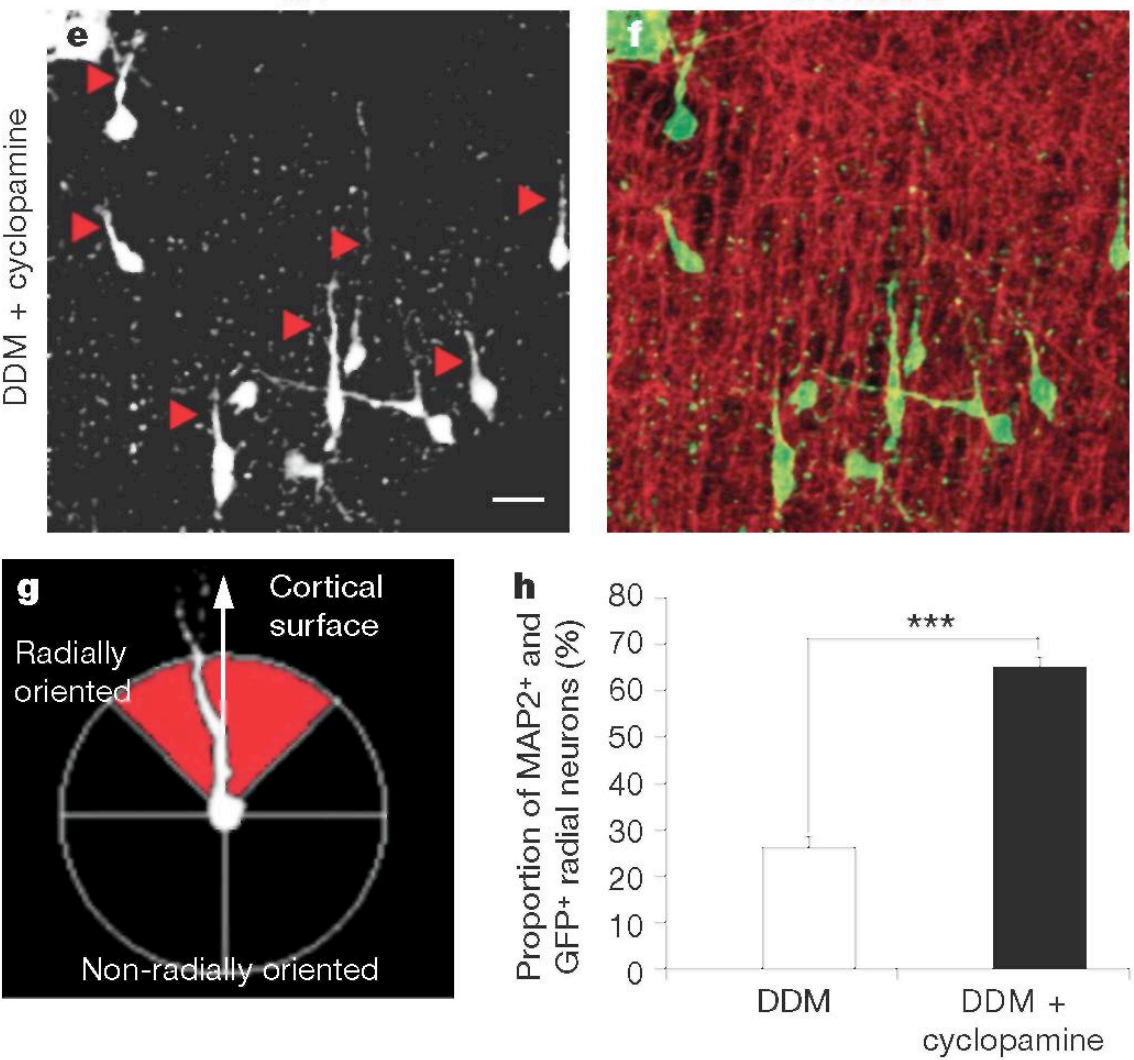


\section{SEQUENTIAL CORTICAL NEUROGENESIS FROM ESCS}

Pyramidal neurons can be subdivided into numerous subtypes, defined in vivo by a repertoire of molecular markers, identifying distinct layer-specific neuronal populations ${ }^{7,22-24}$. To examine the diversity of the neuronal repertoire derived from ESCs in DDM plus cyclopamine, we studied the expression of a large set of layer-specific markers corresponding to the major subtypes of cortical neurons generated in vivo in the mouse (Fig. 4a and Supplementary Fig. 5). We determined the expression of these markers, alone or in combination, at the peak of generation of neurons (day 21) and found that each was expressed by a specific subset of the neurons, according to a combinatorial pattern notably similar to the one observed for native cortical neurons (Supplementary Figs 5 and 6a-i and Supplementary Table 2). Although the repertoire of ESC-derived cortical neurons closely matches the one observed in vivo, we noted some quantitative differences, in particular an under-representation of upper layer subtypes (Fig. 4b, Supplementary Fig. 5 and Supplementary Table 2). This bias is also observed in cultures of genuine cortical progenitors ${ }^{8}$, suggesting that in vivo cues are required for the full generation of upper layer neurons ${ }^{25}$.

These results indicate that a diverse and physiological repertoire of distinct subtypes of neurons corresponding to deep and upper cortical layers are generated from ESCs in DDM plus cyclopamine conditions, raising the question of whether they are specified according to a precise temporal pattern, as observed with native cortical neurons ${ }^{8-22}$ (Fig. 4a). We addressed this issue by examining the timing of onset of expression of these markers throughout the in vitro neurogenesis process (Fig. 4b). The first detectable marker to appear in neurons was reelin, after 6 days of differentiation (Fig. 4b). The early appearance of reelin ${ }^{+}$ neurons suggested that these could correspond to Cajal-Retzius neurons (the earliest generated cortical neurons ${ }^{22}$ ), which was confirmed by combined stainings for other markers of Cajal-Retzius neurons and overlay assays (Supplementary Fig. 7). TBR1 was the second marker to be detected (after 7 days of differentiation), followed by OTX1 (8 days), CTIP2 (9 days) and finally SATB2 and CUX1 (12 days). Overall, this sequence is highly similar to the temporal pattern observed for genuine cortical neurons in vivo and ex vivo (Supplementary Fig. 5) $)^{8,22}$. Next we performed 5-bromodeox-yuridine (BrdU) pulse-chase experiments combined with staining of the neuronal markers to determine the date of birth of each neuronal subtype in the ESC system (Fig. 4c). This analysis revealed that neurons expressing layer-specific markers are generated in sequential overlapping waves: first reelin and TBR $1^{+}$neurons (peak at day 10-11), followed by CTIP2 ${ }^{+}$neurons (peaking at day 12-13), and then by $\mathrm{CUX} 1^{+}$and SATB2 ${ }^{+}$neurons (peaking at day 14-16; Fig. 4c).

\section{Figure 4. The sequential generation of the different subtypes of ESC-derived cortical neurons is similar to the in vivo situation, and is encoded within single cell lineages}

a, Scheme depicting patterns of the layer-specific markers in cortical neurons, and their timing of generation in vivo (adapted from refs 22, 45). b. Evolution in time of the proportion of $\beta$-tubulin- $\mathrm{II}^{+}$neurons expressing layerspecific markers in DDM plus cyclopamine. Coloured arrows indicate the first day of appearance of each marker $(N=3$ experiments), c, Birthdating analysis. Cultures were pulse-labelled with BrdU for $24 \mathrm{~h}$ at various time points (x axis). Cultures were stopped at day 21 , and the proportion of BrdU fully labelled nuclei was quantified among $\beta$ tubulin- $\mathrm{II}^{+}$neurons expressing each specific marker $(N=3$ experiments), d, Clonal analysis: ESC-derived neural progenitors were dissociated after 10 days of differentiation, followed by culture at clonal density for $2-8$ days. The 
proportion of $\beta$-tubulin-III ${ }^{+}$neurons expressing layer-specific markers was then quantified ( $N=3$ experiments). e, Clonal analysis reveals the shift in competence of individual neural progenitors. Neural progenitors from ESCs were dissociated at distinct time points (days 10-16 of differentiation), and cultured for 6 days at clonal density, revealing a shift in competence from early progenitors generating mainly reelin neurons, to later progenitors generating mainly CTIP2 ${ }^{+}$neurons. Values represent the mean number of clones containing at least one marked neuron. $\mathbf{f}, \mathbf{g}$, Typical example of neuronal clones (stained with $\beta$-tubulin-III in green) resulting from dissociation at day 14 followed by 6 days of clonal culture, containing reelin (blue, open arrowhead) and CTIP2 (red, filled arrowhead) neurons (f), and reelin (blue, open arrowhead) and SATB2 (red, arrow) neurons (g). Scale bar represents $20 \mu \mathrm{m}$. All data are represented as mean + s.e.m.

\begin{tabular}{l|l|ll}
\hline \multicolumn{2}{c}{ Layers } & \multicolumn{1}{c}{ Markers } & Birthdate \\
\hline $\begin{array}{l}\text { Cajal- } \\
\text { Retzius } \\
\text { neurons }\end{array}$ & & & \\
\hline & & \\
Upper \\
layers
\end{tabular}

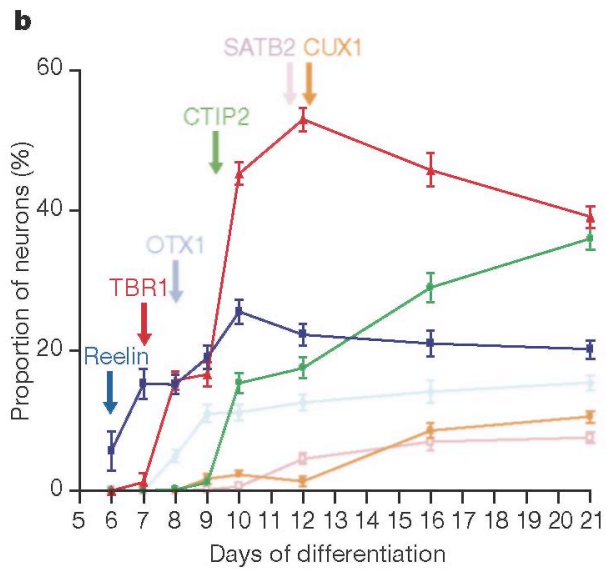

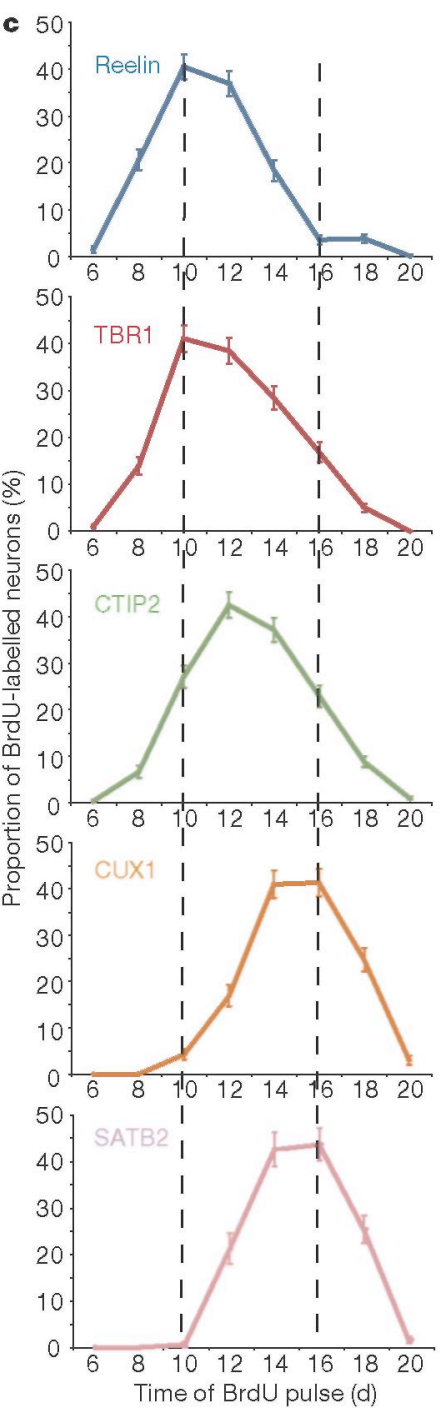
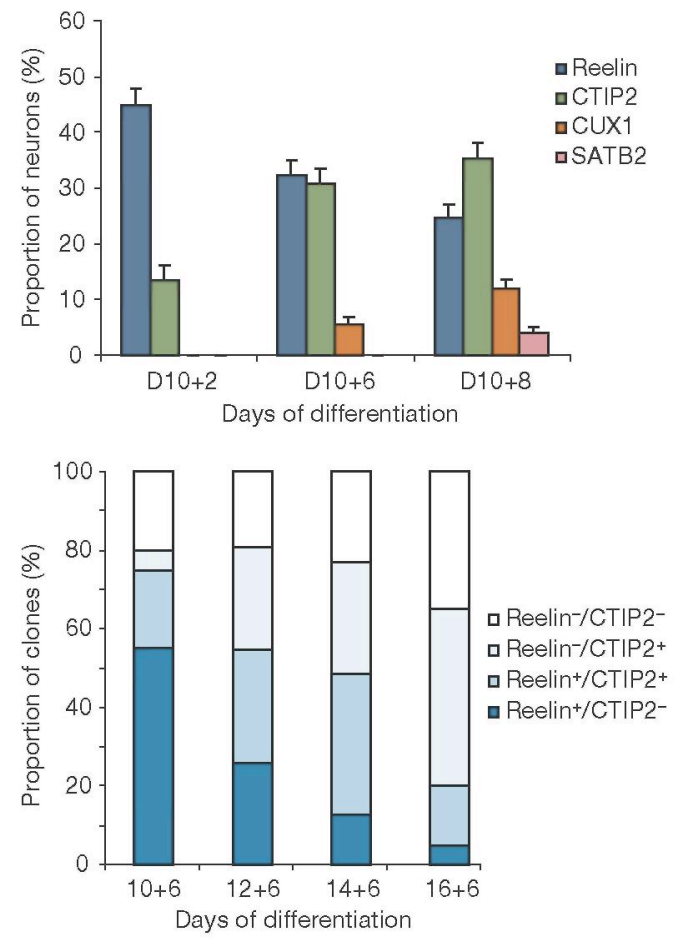

Reelin CTIP2 B-tubulin-II! Reclin SATB2 B-tubulin-1!h
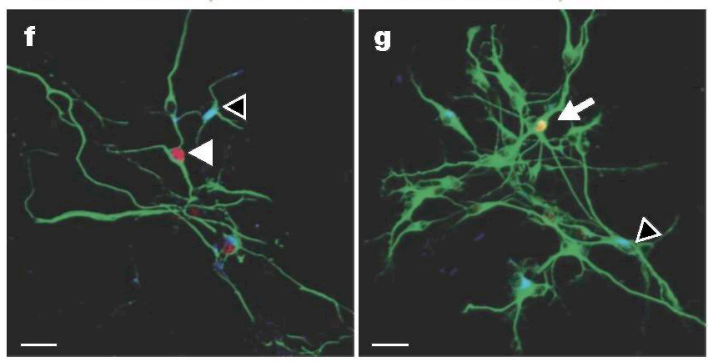

These data demonstrate that ESC-derived neurons corresponding to distinct cortical subtypes appear through a coordinated sequence, as observed in vivo ${ }^{6,26}$. To explore the cellular mechanism involved, we undertook cell lineage experiments using clonal cell analyses ${ }^{8}$. We first performed clonal dilutions of ESC-derived neural progenitors at a fixed starting point (10 days of differentiation) and characterized the neuronal progeny after serial time points (2,6 and 8 days, Fig. $4 d$ ). At early stages ( 2 days of clonal culture), such clones of neural progenitors generated mostly reelin ${ }^{+}$neurons and a few CTIP2 ${ }^{+}$neurons. With time, 
the proportion of reelin ${ }^{+}$neurons decreased substantially, whereas the proportion of CTIP2 ${ }^{+}$ neurons increased. SATB2 ${ }^{+}$and $\mathrm{CUX}^{+}$neurons appeared later in the clones, with a maximum reached after 8 days of clonal culture days in vitro. Next we dissociated neural cultures from ESCs at different time points (from day 10 to day 16) and looked at their progeny after 6 days in vitro (Fig. 4e). Consistently with the previous data, we observed that clones obtained from early progenitors (dissociated at day 10) generated mostly reelin ${ }^{+}$and some CTIP2 $^{+}$neurons after 6 days, whereas clones obtained from later progenitors (dissociated at days 12, 14 and 16) contained fewer and fewer reelin ${ }^{+}$neurons but many more CTIP $2^{+}$neurons. These experiments also enabled detection of a substantial proportion of clones containing different types of neurons, such as reelin and CTIP2 single-labelled neurons (with a maximum at day $14+6$ ) and reelin and SATB2 single-labelled neurons (Fig. $4 \mathrm{e}-\mathrm{g}$ ). Together, these results indicate that the ESC intrinsic pathway leads to the generation of multipotent cortical progenitors that change in competence over time, as suggested for genuine cortical stem cells ${ }^{8,27}$.

\section{CORTICAL AXONAL PROJECTIONS OF ESC NEURONS}

To characterize further the functionality and identity of ESC-derived neurons, we performed in vivo grafting experiments in neonatal mice ${ }^{13,28}$ using a TAU (MAPT)-GFP knock-in ESC line ${ }^{29}$ to identify neurons and their axonal projections. ESCs were allowed to differentiate for 12-17 days in DDM plus cyclopamine conditions, and then were dissociated and grafted into the frontal cortex of mouse neonates. The differentiation and patterns of projections of the grafted cells were analysed after one month (Fig. 5 and Supplementary Fig. 8). In all cases, the graft was localized underneath the frontal cortex or in the grey matter itself, and most of the grafted cells consisted of $\mathrm{GFP}^{+}$neurons that displayed molecular and morphological landmarks of mature pyramidal neurons, with no evidence of teratoma formation (Fig. 5a-d, Supplementary Figs 9 and 10, Supplementary Note 1 and Supplementary Table 3).

We next examined the GFP ${ }^{+}$axonal projections of the grafted neurons, and compared them with the endogenous pattern of cortical projections (schematized in Fig. 5e, f). In most of the grafted animals, we found significant projections throughout the external capsule and corpus callosum, up to the ipsilateral and contralateral cortex, corresponding to cortico-cortical projections (Fig. 5g, h, Supplementary Fig. 8a-c and Supplementary Table 4). GFP ${ }^{+}$ projections corresponding to subcortical efferents were found in the striatum, internal capsule, thalamus, cerebral peduncles and midbrain, and to pontine nuclei in the hindbrain (Fig. 5i-1, Supplementary Fig. 8d, e and Supplementary Table 4). Similar patterns of differentiation and axonal projections were observed when ESC-derived neuronal preparations were grafted in utero in embryonic day 13 embryos (Supplementary Note 2 and Supplementary Fig. 11d-g).

Overall, the projections of the grafted neurons markedly resemble the pattern of cortical efferents, providing further evidence that most neurons generated from ESCs in DDM plus cyclopamine have a cortical identity. These results also demonstrate that the pattern of projections of the neurons is generated by a diverse repertoire of cortical neurons, including projections to the thalamus (corresponding to cortical layer VI), the midbrain and hindbrain (layer V), and the cerebral cortex (layers II/III and V). Importantly, when performing grafting experiments using neurons differentiated in DDM conditions (thus containing far fewer 
pyramidal neurons, Figs 1-3), the grafted neurons displayed fewer cortical-like projections, but instead showed local neurite outgrowth and scattered multipolar neurons (Supplementary Fig. 12 and Supplementary Table 6), confirming that the pattern of projections of neurons derived in DDM plus cyclopamine is mainly the result of an in vitro intrinsic program.

We next examined whether the grafting of ESC-derived neural progenitors and neurons differentiated after different periods of time, and thus possibly enriched for specific laminar fates, could generate different layer-specific patterns of projections in vivo. This analysis revealed that the proportion of projections found in thalamic, midbrain and cortical targets was different depending on the timing of differentiation. Grafts emanating from cells differentiated for a shorter period (12 days) displayed a higher proportion of projections to the thalamus than grafts emanating from cells differentiated for a longer period (days 14-17). Conversely, grafts emanating from later time points (14-17 days) displayed a higher proportion of projections to the cortex than the grafts from earlier time points (12 days), whereas the proportion of projections to the midbrain followed an intermediate temporal pattern (Fig. $5 \mathrm{~m} ; P<0.001, X^{2}$ test). In correlation with the increase in the proportion of cortico-cortical projections for cells cultured for longer periods, the proportion of $\mathrm{CUX} 1^{+}$ neurons, a marker of cortico-cortical projection neurons, was higher in the grafts emanating from cells cultured for 14 days than those cultured for 12 days in vitro ( $3 \pm 1 \%$ versus $11 \pm$ $2 \%$ (mean \pm s.e.m.), $P<0.01$ ). Thus, the intrinsic pathway of corticogenesis leads to the sequential generation of a diverse array of cortical neurons displaying layer-specific patterns of axonal projections.

In addition to layer-specific patterns, cortical neurons display area-specific projections, so that each cortical area projects to selective targets in the rest of the brain $^{30}$ (Fig. 5e, f). To examine this crucial aspect of cortical identity, we performed a detailed analysis of the patterns of projections of ESC-derived grafted neurons. We found that grafted neurons projecting to the cortex, thalamus and mid-brain/hindbrain did not innervate these structures in a diffuse way, but in a precise manner, corresponding mainly to visual and limbic occipital cortex (Fig. 5, Supplementary Fig. 8 and Supplementary Table 4). In the cortex, GFP ${ }^{+}$axons could be seen in most grafted animals in the visual areas (V1/V2), as well as in the limbic cortex, but no projections to motor (MI) or somatosensory (S1) areas were observed in any grafted animal (Fig. $5 \mathrm{n}$ and Supplementary Table 4). In the thalamus (Fig. $5 \mathrm{j}$, $\mathrm{O}$ and Supplementary Table 4), GFP ${ }^{+}$axons were observed to innervate the visual thalamic nuclei in most animals, whereas they were observed to innervate the other primary nuclei of the thalamus in only a few cases. Quantification of the distribution of $\mathrm{GFP}^{+}$fibres in each main thalamic nucleus (Fig. 5o) revealed a much denser innervation in visual thalamic nuclei than in any other primary thalamic nucleus. Similarly, when examining projections to midbrain and hindbrain, in most cases $\mathrm{GFP}^{+}$axons projected to the superior colliculus (Fig. 51, n), the midbrain target of the visual cortex, whereas no fibres could be observed in the pyramidal tract or spinal cord corresponding to motor projections, or to the auditory inferior colliculus. In addition, most grafted animals sent projections to the limbic cortex, medial thalamic nuclei and mid-brain periventricular grey matter, corresponding to structures receiving diffuse projections from the neocortex and limbic cortex (Fig. 5n, Supplementary Fig. 8 and Supplementary Table 4). A similar area-specific pattern of projection was found after different days of in vitro differentiation (days 12-17), suggesting that cells were committed to these specific areal fates after at least 12 days in vitro. 
The projections of ESC-derived neurons look strikingly similar to those described with late embryonic occipital cortex grafted into neonatal frontal cortex (which display a mainly visual identity), but are quite distinct from the pattern of embryonic frontal cortex grafted into frontal cortex (which develop mainly motor-like projections) ${ }^{28,31,32}$. Importantly, our results were all obtained with grafts in the frontal cortex, suggesting that the patterns observed were not due to the re-specification of the grafted neurons through the influence of the host ${ }^{13,31,33}$. ESCderived neural cells thus appear to undergo in vitro an area-specific differentiation process that results in a surprisingly specific identity, corresponding mainly to occipital/visual cortex. Confirming this hypothesis, we found that even before grafting most ESC-derived neural progenitors expressed the CoupTFI (NR2F1) and CoupTFII (NR2F2) transcription factors $(81.64 \pm 2.22 \%$ and $95.1 \pm 1.23 \%$, respectively, Supplementary Fig. $8 \mathrm{f}, \mathrm{g})$, which are preferentially expressed in the embryonic occipital/visual cortex ${ }^{34,35}$.

\section{Figure 5. ESC-derived neurons display a wide range of layer-specific but selective area-specific patterns of neuronal projections when grafted in vivo}

a, Representative case of a GFP ${ }^{+}$(in white) graft located under the cerebral cortex with axonal projections through the internal capsule (small arrows), the external capsule (arrowheads) and the corpus callosum (large arrow), b, c, A graft located within the motor cortex, with most of the cells being GFP ${ }^{+}$(b, in green) and MAP2 ${ }^{+}$ (c, in red), nuclear staining is in blue, d, Single GFP $^{+}$neurons grafted in the cortex display a pyramidal morphology and radial orientation, $\mathbf{e}, \mathbf{f}$, Scheme of the layer- and area-specific endogenous patterns of projections of layer $\mathrm{VI}(\mathbf{e})$ and layer $\mathrm{V}$ (f) neurons, from motor (red), somatosensory (orange), auditory (green) and visual (blue) areas, e, Layer VI neurons project to the thalamus in an area-specific pattern: motor to the ventrolateral (VL), somatosensory to the ventrobasal (VB), auditory to the medial geniculate (MG) and visual to the lateral geniculate (LG) nuclei (other visual targets lateral dorsal (LD) and lateral posterior (LP) not shown), $\mathbf{f}$, Layer $V$ neurons project to more caudal structures in an area-specific pattern: motor to the pediculopontine nuclei (PPN) and the spinal cord, auditory to the inferior colliculus, and visual to the superior colliculus and PPN. g-I, Patterns of projections of the grafted ESC-derived neurons in the corpus callosum ( $\mathbf{g})$, the visual cortex (h), the internal capsule, the striatum (i) and the thalamus (j), with many fibres in LG but very few in the VB (arrow in $\mathbf{j}$ ), the cerebral peduncles $(\mathbf{k})$ and the superior colliculus (arrow, I). PAGM, Periaqueductal gray matter. $\mathbf{m}$, Quantification of the proportion of fibres found in layer-specific targets, from grafted cells differentiated for different time periods in vitro. Increasing the time of differentiation yields more cortico-cortical fibres but less cortico-thalamic fibres $\left({ }^{* * *} P<0.001\right)$. $\mathbf{n}$, Quantification of the proportion of grafted animals displaying $\mathrm{GFP}^{+}$fibres in cortical areas and subcortical targets. IC, inferior colliculus; SpC, spinal cord; SC, superior colliculus. M1, motor area; Pyr, pyramidal tract; SI, somatosensory area; VI, V2, visual areas, o, Quantification of the number of fibres found in selective thalamic nuclei. Area-specific colour codes are the same as in $\mathbf{e}$ and $\mathbf{f}$, and diffuse/ limbic targets are labelled in black $\left({ }^{\star \star *} P<0.001\right)$. All values are displayed as means \pm s.e.m. Scale bars represent 500 $\mu \mathrm{m}(\mathbf{a}), 50 \mu \mathrm{m}$ (b, c, i, k), $20 \mu \mathrm{m}(\mathbf{d})$, or $100 \mu \mathrm{m}(\mathbf{g}, \mathbf{h}, \mathbf{j}, \mathbf{I})$. Dorsal (D) is up and medial (M) is left. 

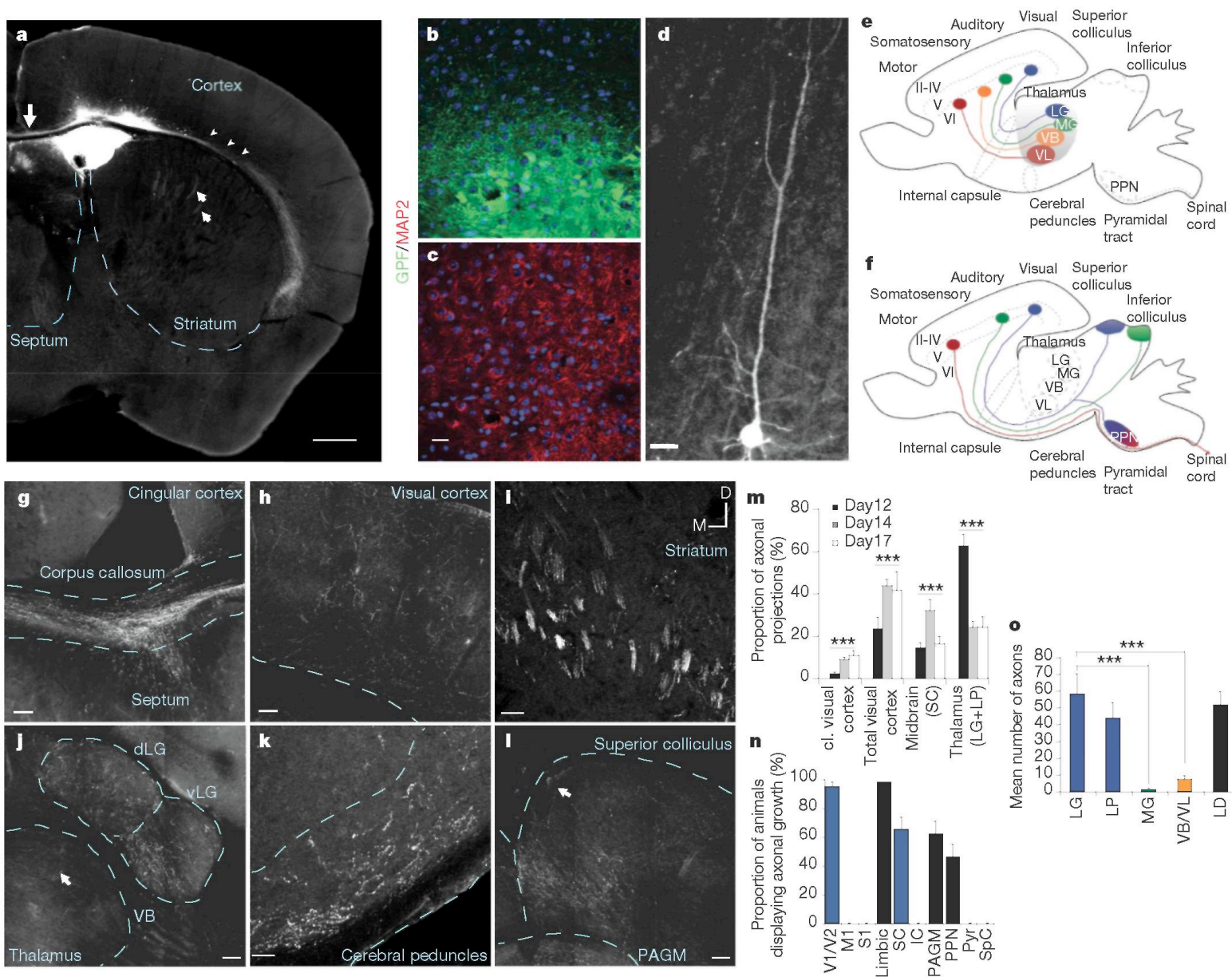

\section{DISCUSSION}

ESCs constitute a powerful resource in neurobiology, enabling the directed specification of spinal, hindbrain and midbrain neurons ${ }^{36-38}$. The ability for mouse ESCs to generate forebrain-like progenitors was reported previously, but the identity of the neurons generated had remained uncertain ${ }^{39-41}$. Here we demonstrate that ESCs can be efficiently specified into a repertoire of neurons that display cardinal features of cortical neurons, including patterns of axonal projections, opening new avenues to apply ESC technology to cortical biology and diseases ${ }^{42}$.

Corticogenesis from ESCs follows the major milestones of cortical development, including regional and temporal patterning of pyramidal neuron specification, through the generation of multipotent cortical progenitors that sequentially produce neurons displaying distinct layerspecific identities. In contrast, ESC-derived cortical progenitors have a much more restricted capacity to develop diverse area-specific identities, as most of the generated neurons display a selective pattern of visual/limbic areas. This surprising finding indicates that a specific cortical area can differentiate without any influence from the brain, through an intrinsic pathway that may correspond to an ancestral developmental program of the mammalian forebrain $^{43,44}$. 


\section{METHODS SUMMARY}

Embryonic stem cells were propagated using standard procedures. For neural differentiation, ESCs were plated at low density on gelatin-coated dishes and cultured in DDM, devoid of any morphogen or serum derivative. Cyclopamine was added from day 2 to day 10 of differentiation. After 10-14 days of differentiation, neural progenitors were dissociated and plated on polylysine/laminin and cultured in N2B27 medium. For clonal analyses, neural progenitors were dissociated and cultured on polylysin/laminin-coated coverslips at clonal density. Immunofluorescent and RT-PCR detection of the markers were performed according to standard methods. Pyramidal morphology analysis and overlay assays were performed as described $^{20,21}$, and electrophysiology was performed using standard procedures. In vivo grafting was performed as described for embryonic cortical neurons ${ }^{31}$ using neonatal or embryonic mice.

Full Methods and any associated references are available in the online version of the paper at www.nature.com/nature.

\section{References}

1. Wilson, S. W. \& Houart, C. Early steps in the development of the forebrain. Dev. Cell 6,167-181 (2004).

2. Gunhaga, L, Jessell, T. M.\& Edlund, T. Sonic hedgehog signaling at gastrula stages specifies ventral telencephalic cells in the chick embryo. Development 127, 3283-3293 (2000).

3. Sur, M. \& Rubenstein, J. L. Patterning and plasticity of the cerebral cortex. Science 310,805-810(2005).

4. Schuurmans, C. \& Guillemot, F. Molecular mechanisms underlying cell fate specification in the developing telencephalon. Curr. Opin. Neurobioi. 12, 26-34 (2002).

5. Gotz, M. \& Sommer, L. Cortical development: the art of generating cell diversity. Development 132, 3327-3332 (2005).

6. Bayer, S. \& Altman, J. Neocorticoi Development (Raven Press, 1991).

7. Molyneaux, B. J., Arlotta, P., Menezes, J. R. \& Macklis, J. D. Neuronal subtype specification in the cerebral cortex. Nature Rev. Neurosci. 8, 427-437 (2007).

8. Shen, Q. et al. The timing of cortical neurogenesis is encoded within lineages of individual progenitor cells. Nature Neurosci. 9, 743-751 (2006).

9. McConnell, S. K. \& Kaznowski, C. E. Cell cycle dependence of laminar determination in developing neocortex. Science 254, 282-285 (1991).

10. Frantz, G. D. \& McConnell, S. K. Restriction of late cerebral cortical progenitors to an upper-layer fate. Neuron 17, 55-61 (1996). 
11. Vanderhaeghen, P. \& Polleux, F. Developmental mechanisms patterning thalamocortical projections: intrinsic, extrinsic and in between. Trends Neurosci. 27, 384-391 (2004).

12. Rash, B. G. \& Grove, E. A. Area and layer patterning in the developing cerebra cortex. Curr. Opin. Neurobioi. 16, 25-34 (2006).

13. O'Leary, D. D., Chou, S. J. \& Sahara, S. Area patterning of the mammalian cortex. Neuron 56, 252-269 (2007).

14. Ying, Q. L, Stavridis, M., Griffiths, D., Li, M. \& Smith, A. Conversion of embryonic stem cells into neuroectodermal precursors in adherent monoculture. Nature Biotechnol. 21,183186 (2003).

15. Smukler, S. R., Runciman, S. B., Xu, S., \&. van der Kooy. D. Embryonic stem cells assume a primitive neural stem cell fate in the absence of extrinsic influences. J. Cell Biol. 172, 79-90(2006).

16. Munoz-Sanjuan, I. \& Brivanlou, A. H. Neural induction, the default model and embryonic stem cells. Nature Rev. Neurosci. 3, 271-280 (2002).

17. Qian, X. et al. Timing of CNS cell generation: a programmed sequence of neuron and glial cell production from isolated murine cortical stem cells. Neuron 28, 69-80 (2000).

18. Chen, J. K., Taipale, J., Cooper, M. K. \& Beachy, P. A. Inhibition of Hedgehog signaling by direct binding of cyclopamine to Smoothened. Genes Dev. 16, 2743-2748 (2002).

19. Inoue, T., Nakamura, S. \& Osumi, N. Fate mapping of the mouse prosencephalic neural plate. Dev. Biol. 219, 373-383 (2000).

20. Hand, R. et al. Phosphorylation of Neurogenin2 specifies the migration properties and the dendritic morphology of pyramidal neurons in the neocortex. Neuron 48, 45-62(2005).

21. Polleux, F., Morrow, T. \& Ghosh, A. Semaphorin 3A is a chemoattractant for cortical apical dendrites. Nature 404, 567-573 (2000).

22. Hevner, R. F. et al. Beyond laminar fate: toward a molecular classification of cortical projection/pyramidal neurons. Dev. Neurosci. 25,139-151 (2003).

23. Britanova, O. et al. Satb2 is a postmitotic determinant for upper-layer neuron specification in the neocortex. Neuron 57, 378-392 (2008).

24. Alcamo, E. A. et al. Satb2 regulates callosal projection neuron identity in the developing cerebral cortex. Neuron 57, 364-377 (2008).

25. Dehay, C. \& Kennedy, H. Cell-cycle control and cortical development. Nature Rev. Neurosci. 8, 438-450 (2007).

26. Hevner, R. F. From radial glia to pyramidal-projection neuron: transcription factor cascades in cerebral cortex development. Moi. Neurobioi. 33, 33-50 (2006).

27. Noctor, S. C, Martinez-Cerdeno, V., Ivic, L. \& Kriegstein, A. R. Cortical neurons arise in symmetric and asymmetric division zones and migrate through specific phases. Nature Neurosci. 7,136-144 (2004). 
28. Gaillard, A., Gaillard, F. \& Roger, M. Neocortical grafting to newborn and adult rats: developmental, anatomical and functional aspects. Adv. Anat. Embryo!. Ceil Biol. 148,186(1998).

29. Wernig, M. et al. Tau EGFP embryonic stem cells: an efficient tool for neuronaineage selection and transplantation. J. Neurosci. Res. 69, 918-924 (2002).

30. Paxinos, G. The Rat Nervous System (Academic, 1995).

31. Pinaudeau, C, Gaillard, A. \& Roger, M. Stage of specification of the spina I cord and tectal projections from cortical grafts. Eur. J. Neurosci. 12, 2486-2496 (2000).

32. Ebrahimi-Gaillard, A., Guitet, J., Gamier, C. \& Roger, M. Topographic distribution of efferent fibers originating from homotopic or heterotopic transplants: heterotopical ly transplanted neurons retain some of the developmental characteristics corresponding to their site of origin. Brain Res. Dev. Brain Res. 77, 271-283 (1994).

33. Barbe, M. F. \& Levitt, P. Age-dependent specification of the corticocortica connections of cerebral grafts. J. Neurosci. 15,1819-1834 (1995).

34. Armentano, M. et al. COUP-TFI regulates the balance of cortical patterning between frontal/motor and sensory areas. Nature Neurosci. 10,1277-1286 (2007).

35. Sansom, S. N. et al. Genomic characterisation of a Fgf-regulated gradient-based neocortical protomap. Development 132, 3947-3961 (2005).

36. Lee, S. H., Lumelsky, N., Studer, L, Auerbach, J. M. \& McKay, R. D. Efficient generation of midbrain and hindbrain neurons from mouse embryonic stem cells. Nature Biotechnol. 18, 675-679 (2000).

37. Wichterle, H., Lieberam, I., Porter, J. A. \& Jessell, T. M. Directed differentiation of embryonic stem cells into motor neurons. Ceil 110, 385-397 (2002).

38. Andersson, E. et al. Identification of intrinsic determinants of midbrain dopamine neurons. Cell 124, 393-405 (2006).

39. Bibel, M. et al. Differentiation of mouse embryonic stem cells into a defined neuronal lineage. Nature Neurosci. 7,1003-1009 (2004).

40. Glaser, T. \& Brustle, O. Retinoic acid induction of ES-cell-derived neurons: the radial glia connection. Trends Neurosci. 28, 397-400 (2005).

41. Watanabe, K. et al. Directed differentiation of telencephalic precursors from embryonic stem cells. Nature Neurosci. 8, 288-296 (2005).

42. Gaillard, A. et al. Reestablishment of damaged adult motor pathways by grafted embryonic cortical neurons. Nature Neurosci. 10,1294-1299 (2007).

43. Puelles, L. Thoughts on the development, structure and evolution of the mammalian and avian telencephalic pallium. Phil. Trans. R. Soc. Lond. B 356, 1583-1598(2001).

44. Frost, S. B., Milliken, G. W., Plautz, E. J., Masterton, R. B. \& Nudo, R. J. Somatosensory and motor representations in cerebral cortex of a primitive mammal (Monodeiphis domestica): a window into the early evolution of sensorimotor cortex. J. Comp. Neurol. 421, 29-51 (2000). 
45. von Economo, C. \& Koskinas, G. The Cytoarchitectonics of the Adult Human Cortex (Springer, 1925).

Supplementary Information is linked to the online version of the paper at www.nature.com/nature.

\section{Acknowledgements}

We thank G. Vassart, M. Pandolfo and members of the laboratory and IRIBHM for support and discussions. We are indebted to A. Bilheu for technical assistance, J.-M. Vanderwinden for help with confocal microscopy, and V. De Maertelaer for statistical analyses. We thank F. Polleux, B. Hassan and C. Blanpain for comments on the manuscript. We are grateful to $S$. Arber, A. Goffinet, R. Hevner, R. di Lauro, Y. Sasai, S.Stifani, M. Studer and V. Tarabykin for providing us with antibodies, and to Y.-A. Barde for providing Tau-GFP ESC lines. This work was funded by the Belgian FN RS, the Action de Recherches Concertées (ARC) Programs (to P.V. and S.N.S.), the Interuniversity Attraction Poles Program (IUAP), Belgian State, Federal Office, the Walloon Region Excellence Program CIBLES, the Belgian Queen Elizabeth Medical Foundation and a UCB Neuroscience Award (to P.V.), the Tournesol FNRS/CNRS Program (to P.V. and A.G.), Télévie (to S.N.S.), and a Marie Curie Grant (to T.B.). P.V. is a Senior Research Associate of the FNRS, and N.G., R.H., T.B., J.D. and LP. were funded as Research Fellows of the FNRS.

\section{METHODS}

ESC culture. Embryonic stem cells (E14Tg2a (obtained from Baygenomics), E14Tg2a-GFP, a derivative thereof, and the TAU-GFP knock-in line ${ }^{29}$ ) were routinely propagated using standard procedures ${ }^{14}$ on gelatin-coated cell culture plastic dishes $(0.1 \%$ gelatin, Sigma; for E14Tg2a cell lines) or on mitotically inactivated mouse embryonic fibroblasts (MEFs; for the TAU-GFP KI cell line).

Neural differentiation. The neural differentiation protocol was adapted from the adherent monoculture protocol described previously ${ }^{14}$. Similar results were obtained with several ESC lines, including distinct derivatives of E14 and J1 cells. ESCs were trypsinized, dissociated and plated at a density of $5 \times 10^{3}$ cells per $\mathrm{cm}^{2}$ on gelatin-coated cell culture plastic dishes in ESC medium. After adhesion, medium was changed to DDM. DDM consists of DMEM/F12 (Invitrogen-Gibco) supplemented with 1XN2 supplement (100X N2 supplement consists of $8.61 \mu \mathrm{M}$ insulin, $1 \mathrm{mM}$ transferrin, $2 \mu \mathrm{M}$ progesterone, $10.01 \mathrm{mM}$ putrescine and $3.01 \mu \mathrm{M}$ selenite; Invitrogen-Gibco), $2 \mathrm{mM}$ glutamine, $1 \times$ MEM-nonessential amino acids, $1 \mathrm{mM}$ sodium pyruvate, $0.5 \mathrm{mg} \mathrm{ml}^{-1}$ bovine serum albumin (BSA) fraction $\mathrm{V}$ (all from InvitrogenGibco), and $110 \mu \mathrm{M} \beta$-mercaptoethanol (Sigma). Cyclopamine (Calbiochem) was added from day2 to day 10 in the differentiation medium at a final concentration of $1 \mu \mathrm{M}$. After 10 to 14 days of differentiation, cells were trypsinized, dissociated and plated on polylysine/laminin 
(Becton-Dickinson) coated glass coverslips and allowed to grow for 4-14 days in N2B27 medium. N2B27 medium consists of a 1:1 mixture of DMEM/F12 supplemented with $1 \mathrm{X}$ N2, $2 \mathrm{mM}$ glutamine, $0.5 \mathrm{mg} \mathrm{ml}^{-1} \mathrm{BSA}$ fraction $\mathrm{V}$ and $110 \mu \mathrm{M} \beta$-mercatoethanol with Neurobasal supplemented with B27 (without vitamin A; Invitrogen-Gibco) and $2 \mathrm{mM}$ glutamine.

Immunofluorescence and quantification. Medium was removed and cells were rinsed with PBS and then fixed for 30min in $4 \%$ paraformaldehyde (PFA) in PBS, $\mathrm{pH} 7.4$, at $4{ }^{\circ} \mathrm{C}$ PFA was removed and cells were rinsed with PBS. Blocking and permeabilization was done in PBS with 5\% horse serum (Invitrogen-Gibco), 3\% BSA (Sigma) and 0.3\% Triton X-100 (Sigma). Incubation with primary antibodies was done in PBS with $1 \%$ horse serum, 3\% BSA and $0.1 \%$ Triton $\mathrm{X}-100$ overnight $(12-18 \mathrm{~h})$ at $4^{\circ} \mathrm{C}$ Cells were rinsed three times with PBS and incubated with secondary antibodies in PBS with $1 \%$ horse serum, $33 \%$ BSA and $0.1 \%$ Triton X-100 for one hour at room temperature $\left(18-20^{\circ} \mathrm{C}\right)$, and then rinsed again with PBS. For multiple labellings, the process was repeated. Nuclei were stained with bisbenzimide (Hoechst 33258; Sigma). For some antibodies, antigen retrieval was used before blocking. The primary and secondary antibodies used are described in Supplementary Methods 1.

For quantification of the proportion of cells expressing a specific marker, at least 300 cells from three independent experiments (at least 100 cells from each experiment) were counted for each time point studied.

BrdU labelling. For pulse-chase experiments, BrdU (Becton Dickinson) was added to the differentiation medium on the different days (see text) during $24 \mathrm{~h}$ at a final concentration of $10 \mu \mathrm{M}$. Cells were dissociated after 21 days of differentiation and plated on polylysin/laminincoated coverslips for an additional day. For short-term BrdU uptake experiments, BrdU was added to the differentiation medium during $1 \mathrm{~h}$ before cell dissociation and replating. BrdU was detected by immunofluorescence as described above.

Clonal analyses. ESC-derived neural progenitors at day 10-16 were trypsinized and thoroughly dissociated with a Pasteur pipette and subsequently with a $200-\mu$ lip into a singlecell suspension. Dissociation was checked under a brightfield microscope and usually yields $>99 \%$ single cells. Cells were plated on polylysin/ laminin-coated coverslips at clonal density (3-5 cells per $\mathrm{mm}^{2}$ ) into clonal medium supplemented with fibroblast growth factor (FGF2). Clonal medium was adapted from ref. 8 and consists in Neurobasal supplemented with $1 \mathrm{X}$ $\mathrm{N} 2,1 \mathrm{X}$ B27, 2mM glutamine and 1X penicillin-streptomycin (Gibco). Half of the medium was changed every three days. FGF2 was added to the medium at concentrations $\left(0.1 \mathrm{ng} \mathrm{ml}^{-1}\right.$ or $10 \mathrm{ng} \mathrm{ml}^{-1}$ ) that have been shown to sustain clonal growth from single cortical progenitors and to allow the sequential generation of the different populations of cortical neurons ${ }^{8}$.

RT-PCR. RT-PCR was performed according to Supplementary Methods 2.

Morphology. For the morphology study, we used the PMI described previously ${ }^{20}$. Pictures of at least 100 neurons stained for MAP2 were taken randomly for each condition and from two different experiments, and the quantification was done blindly in Adobe Photoshop Software using a $25 \mu \mathrm{m}$ circle drawn on a superposed layer.

Overlay assay. Postnatal day 2 mice brains were dissected in cold L15 buffer supplemented with glucose, embedded in $3 \%$ low-melting point agarose in L15, and sectioned (coronal) on a vibratome at $300 \mu \mathrm{m}$. Slices were cultured on a transparent porous membrane ( $1 \mu \mathrm{m}$ pore size, Greiner) in a 35-mm well containing N2B27 medium. Neurons derived from GFP- 
expressing ESCs were dissociated after 21 days by trypsinization and mechanical trituration, washed and resuspended at $1 \times 10^{6}$ cells per millilitre before being plated onto cortical slices. Slices were cultured for 4 days after plating, and then fixed with $4 \%$ PFA overnight at $4{ }^{\circ} \mathrm{C}$ and processed for immunofluorescence. PFA was removed and slices were rinsed three times with PBS. Immunofluorescence was performed as described previously. Quantification of radial dendrite orientation was done blindly according to ref. 21 using Adobe Photoshop software. At least 300 cells on at least 9 different slices coming from 3 independent experiments were assessed (at least 100 cells from each experiment).

Electrophysiology. Electrophysiological recordings were performed according to standard procedures (Supplementary Methods 3).

In vivo grafting experiments. In vivo grafting in neonatal (day of birth or one day later) mice of ESC-derived cortical neurons (after 12-17 days in DDM plus cyclopamine or DDM) was performed as described previously for genuine embryonic cortical neurons ${ }^{31}$, with modifications described in Supplementary Methods 4. In utero grafting experiments were performed according to Supplementary Methods 5. Analysis and quantification of the grafts and their projections are described in Supplementary Methods 6. 